\title{
Protein complex prediction with AlphaFold-Multimer
}

\author{
Richard Evans $^{1^{*}}$, Michael O'Neill ${ }^{1 *}$, Alexander Pritzel ${ }^{1 *}$, Natasha Antropova ${ }^{1 *}$, Andrew Senior $^{1}$, Tim Green $^{1}$, \\ Augustin Žídek $^{1}$, Russ Bates ${ }^{1}$, Sam Blackwell ${ }^{1}$, Jason Yim ${ }^{1}$, Olaf Ronneberger ${ }^{1}$, Sebastian Bodenstein ${ }^{1}$, Michal \\ Zielinski $^{1}$, Alex Bridgland ${ }^{1}$, Anna Potapenko ${ }^{1}$, Andrew Cowie ${ }^{1}$, Kathryn Tunyasuvunakool ${ }^{1}$, Rishub Jain ${ }^{1}$, Ellen \\ Clancy $^{1}$, Pushmeet Kohli ${ }^{1}$, John Jumper ${ }^{1^{*}}$ and Demis Hassabis ${ }^{1^{*}}$ \\ ${ }^{1}$ DeepMind, London, UK, "These authors contributed equally
}

While the vast majority of well-structured single protein chains can now be predicted to high accuracy due to the recent AlphaFold [1] model, the prediction of multi-chain protein complexes remains a challenge in many cases. In this work, we demonstrate that an AlphaFold model trained specifically for multimeric inputs of known stoichiometry, which we call AlphaFold-Multimer, significantly increases accuracy of predicted multimeric interfaces over input-adapted single-chain AlphaFold while maintaining high intra-chain accuracy. On a benchmark dataset of $\mathbf{1 7}$ heterodimer proteins without templates (introduced in [2]) we achieve at least medium accuracy (DockQ $[3] \geq 0.49$ ) on 14 targets and high accuracy (DockQ $\geq 0.8$ ) on 6 targets, compared to 9 targets of at least medium accuracy and 4 of high accuracy for the previous state of the art system (an AlphaFold-based system from [2]). We also predict structures for a large dataset of 4,433 recent protein complexes, from which we score all non-redundant interfaces with low template identity. For heteromeric interfaces we successfully predict the interface (DockQ $\geq 0.23$ ) in $67 \%$ of cases, and produce high accuracy predictions (DockQ $\geq 0.8$ ) in $23 \%$ of cases, an improvement of +25 and +11 percentage points over the flexible linker modification of AlphaFold [4] respectively. For homomeric interfaces we successfully predict the interface in $69 \%$ of cases, and produce high accuracy predictions in $34 \%$ of cases, an improvement of +5 percentage points in both instances.

\section{Introduction}

The formation of permanent and transient protein complexes underpins most biological processes, and understanding the structure of these complexes is a key step towards understanding and modifying their function. In silico protein structure prediction is playing an increasingly important role in biological research and applications as it has approached experimental accuracy, with the recent structure prediction system AlphaFold [1] providing a step change in structure prediction accuracy. Though AlphaFold was trained on individual protein chains, including many proteins whose structure was solved in complex with other proteins, it has shown remarkable ability to predict protein structures with bound co-factors or proteins stabilised by their multimeric interactions. Subsequent work has also shown that providing pseudo-multimer inputs (e.g. residue gap insertion or chains joined with a flexible linker) to the singlechain AlphaFold model is often successful at predicting multimer interactions $[4,5,6,7,8,9]$. These papers have shown surprising generalization performance of the original trained AlphaFold model but leave open the question of how much more accurate AlphaFold is when training is also adapted for multimeric inputs.

The AlphaFold system has recently been described in publication [1] along with source code and model parameters [10]. To summarize briefly, it combines information from the amino acid sequence, multiple sequence alignments and homologous structures in order to predict the structure of individual protein chains. The core part of the neural network, called Evoformer, consists of a neural representation of the multiple sequence alignment (MSA) and pairwise relations between the different amino acids in the protein. These two representations are mixed and processed by a collection of neural network 
modules. The pair representation can be thought of as containing information about the relative positions of amino acids in the chain. This representation is used to predict the relative distances between the amino acids in the chain via a binned distance distribution (distogram). The first row of the MSA embedding is then used together with the pair embedding to predict the final structure. The model is trained end-to-end with gradients propagating from the predicted structure through the entire network.

In this work we extend AlphaFold to multiple chains during both training and inference, with native support for multi-chain featurization and symmetry handling. We refer to this system as AlphaFoldMultimer and demonstrate superior performance compared to existing approaches, including those based on using AlphaFold.

\section{Methods}

Multiple changes to the AlphaFold system were made to adapt it to training on protein complexes, which are detailed below. Summarizing briefly, we modify the losses to take into account permutation symmetry among identical chains, pair the MSA alignments between individual chains to surface cross-chain genetic information, introduce a new way of selecting subsets of residues for training, and make various small adjustments to the structure losses and the model architecture.

\subsection{Multi-Chain Permutation Alignment}

When computing the losses and scoring homomeric components, permutation symmetry must be taken into account. When a protein of a given sequence appears multiple times in the complex, the mapping between the predicted and the ground-truth coordinates is arbitrary and so the model cannot be assumed to predict chains in the same order as in the ground truth. To account for this we aim to pick the optimal permutation of predicted homomer chains that best matches the ground truth. The complexity of optimizing over all permutations grows combinatorially so we employ a simple heuristic that greedily tries to find a good permutation. The details of this heuristic are explained in subsection 7.3.

\subsection{Cross-Chain Genetics}

It has been shown that pairwise correlation in a Multiple Sequence Alignment (MSA) is a useful feature in predicting contacts and 3D structure, but this requires determination of interacting homologs in the heteromeric case, which is in general ambiguous [11]. In this work we provide explicit aligned sequences to the network following the procedure of Zhou et al. [12]. We pair sequences using the UniProt species annotation and resolve ambiguities using the following procedure. If the target protein is prokaryotic, the within-species pairing is done using the smallest genetic distance (approximated by the difference between their UniProt accession IDs). If the target protein is eukaryotic, we rank the candidate rows of each chain by similarity to their respective target sequence, and concatenate pairs of the same rank. More details are available in subsection 7.4.

\subsection{Multi-Chain Cropping}

The number of residues that AlphaFold-Multimer can be trained on is limited by memory and compute considerations as both memory use and compute use increase rapidly with the total number of amino acids in the complex. To ameliorate this, the AlphaFold system is trained on cropped segments of full length proteins, where these cropped regions are contiguous blocks of residues up to 384 residues.

We modified this procedure when training on complexes as the cropped regions need to involve multiple chains in a given complex, and binding interfaces between chains are crucial for modelling 
protein complexes. Therefore, we designed a cropping procedure that maximizes chain coverage and crop diversity whilst ensuring good balance between interface and non-interface regions. We balance per-chain contiguous cropping in sequence space with interface biased spatial cropping in coordinate space. The details of this procedure are described in subsection 7.2.

\subsection{Architecture and Losses}

AlphaFold uses a Frame Aligned Point Error (FAPE) loss [1], whereby the distances between ground truth and predicted atoms are computed in the local reference frame of every residue. In AlphaFold, this loss was clamped at $10 \AA$. For training the multimer model we made changes to the loss function used: for the intra-chain amino acid pairs of the complex we keep the same $10 \AA$ clamping; for the inter-chain pairs we use an unclamped FAPE loss. This provides a better gradient signal for incorrect interfaces. Moreover, we add extra positional encodings denoting whether a given pair of amino acids corresponds to different chains and whether they belong to different homomer or heteromer chains.

We also made various small changes to the model and implementation in order to facilitate inference of larger proteins for a given amount of memory. The details of these, and all other modifications mentioned in this section, are explained in the Supplementary Information.

\subsection{Training Regimen}

AlphaFold-Multimer was trained in a very similar way to AlphaFold [1]. The training dataset comprised structures from the Protein Data Bank (PDB) [13] with a maximum release date of 2018-04-30. Chains were sampled in inverse proportion to cluster size and their corresponding mmCIFs selected as input to the data pipeline, meaning that the rest of the chains in the bio-assembly will be included. This means that the probability of sampling a particular PDB entry is proportional to the sum of probabilities of the individual chain clusters for all chains in the file. The chain clusters are $40 \%$ identity clusterings of the Protein Data Bank with MMSeqs2 [14].

We randomly crop to 384 residues according to the sampling procedure outlined in subsection 2.3 . We train the model to convergence (approximately 10M samples, for 2 weeks) across 128 TPUv3 cores with a batch size of 1 per TPU core. Then we halve the learning rate and double the number of sequences fed into the MSA stack before running two separate fine-tuning stages (one further day of training each), the first with the experimentally resolved and predicted LDDT (pLDDT) heads switched on, and the second with violation losses enabled. These extra heads and losses are identical to those used in the AlphaFold paper [1]. We trained 3 models for the first stage; the best model on the validation set was then fine tuned with 5 different random seeds for the two fine-tuning stages, producing 5 models in total.

\subsection{Inference Regimen}

As with AlphaFold [1], at inference time we run all 5 trained models and select the best model on each target according to model confidence. The only difference to AlphaFold is that our model confidence metric is slightly modified to upweight accuracy on interfaces, as described in subsection 2.7. The inference times for different sequence lengths are similar to those reported in [1].

\subsection{Model Confidence}

The AlphaFold model provides intrinsic model accuracy estimates in the form of a predicted TM-score (pTM) [1, §1.9.7]. We provide a similar metric for the AlphaFold-Multimer model, but modified to score 
interactions between residues of different chains, since we are predominantly interested in the accuracy of interfaces. We call this metric Interface pTM, or ipTM. See subsection 7.9 for more details.

In practice we use a weighted combination of PTM and ipTM as our model confidence metric, in order to account for some intra-chain confidence in our model ranking:

$$
\text { model confidence }=0.8 \cdot \mathrm{ipTM}+0.2 \cdot \mathrm{pTM}
$$

\section{Related Work}

Traditional approaches to multimeric structure prediction have tended to rely on a hybrid of templatebased modelling and free docking $[15,16,17,18]$. Template-based modelling approaches search for homologous complexes of known structure to serve as template models for the system under study. Free docking approaches start from individual subunits of known structure and attempt to select the most plausible interfaces from thousands of sampled orientations. The top 3 entrants of CASP14 Multimers Baker-experimental [19], Venclovas [20] and Takeda-Shitaka lab [21] — all used methodologies based on these approaches. Takeda-Shitaka used a purely template-based approach, whilst Baker-experimental and Venclovas used a combination of template-based and free-docking. Baker-experimental additionally employed a deep learning based system to infer inter-chain contacts from co-evolution information, which were used to guide the overall sampling and scoring of docked structures.

Inspired by AlphaFold's recent success at CASP14, there have now been a number of attempts to apply the trained AlphaFold network to the task of complex structure prediction. Many of these approaches simply add a gap or linker segment between chains of a complex, and then fold it with pre-trained AlphaFold as if it were a single chain $[4,5,6,7,8,9]$, the residue gap trick having been previously applied to RoseTTAFold [22]. The idea is that the neural network should identify the linker segment as unstructured and fold the single multi-domain chain in a similar way to multiple chains. Bryant et al. [9] benchmarked AlphaFold with a residue gap against RoseTTAFold and the rigid-docking method GRAMM [23], and AlphaFold substantially outperformed both other methods. Ghani et al. [2] combine this approach with docking using the ClusPro docking server [17]. Humphreys et al. [24] used AlphaFold with RoseTTAFold pre-screening to generate a dataset of 712 high confidence complexes, with previously unknown structure. All of these approaches use a single-chain trained AlphaFold or AlphaFold-like system at inference time; predictions on complexes are achieved simply by modifying the input. In this work we natively handle multimer chains at both training and inference time, and our system is trained on a dataset that includes multimers.

A key aspect of the AlphaFold model is its ability to learn to identify residue conservation and co-evolution directly from the MSA. Such an ability has also been shown to be a useful factor for predicting protein-protein interactions $[25,26]$. However, constructing an informative multi-chain MSA is a challenging task, often involving the careful pairing of sequences between chains in order to construct valid interlogs of the target sequence that expose interface information. Various heuristic methods exist to perform this pairing [27, 28, 29, 12]. We follow the approach of Zhou et al. [12] by pairing by species label, disambiguating using genetic distance for prokaryotes, and similarity to the target sequence for eukaryotes. Note that [5] only performs pairing for prokaryotes (using genetic distance), whilst [9] doesn't distinguish between prokaryotes and eukaryotes, disambiguating in both cases with the similarity-based method.

\section{Results}

AlphaFold-Multimer predictions are generated as described in subsection 2.6. We benchmarked AlphaFoldMultimer on two datasets: Benchmark 2 from [2], and Recent-PDB-Multimers. Benchmark 2 is a set 
of 17 heterodimers from PDB after the training set cutoff date. The authors selected the targets such that there are no homologous complexes in PDB. Recent-PDB-Multimers is a homology-reduced set of 4,433 recent protein complexes from PDB. Predictions were made on full complexes before splitting into in-contact chain pairs (according to the ground truth) for analysis. These pairs were clustered to remove redundancy and filtered so that neither chain in the pair had greater than $40 \%$ template identity to the training set, yielding 2,603 unique pairs upon which we report metrics. For details see subsection 7.8. For both datasets we compare models using DockQ score $[3,30]$. DockQ measures the quality of the interface and yields a score in the range $[0,1]$, interfaces with score $<0.23$ are considered incorrect and scores $>0.8$ are of the highest quality.

\subsection{Benchmark 2}

On Benchmark 2 we compare AlphaFold-Multimer to the following systems:

- AlphaFold-Linker: In this setup we add a 21 residue repeated Glycine-Glycine-Serine linker between each chain before running it as a single chain through the AlphaFold model. Similar linker modifications to AlphaFold have previously been used [4].

- AlphaFold-Gap (ColabFold [5]): This is a third-party Google Colab, version from 2021-08-16, that runs AlphaFold with a 200 residue gap in the residue index between chains. It uses MMSeqs2 [14] for genetics, includes MSA pairing and doesn't include templates.

- ClusPro: This setup runs AlphaFold on the individual chains before docking them together using ClusPro.

- AlphaFold refined ClusPro: The ClusPro predictions are refined by feeding them back into the AlphaFold model as templates. The resulting 10 predictions are re-ranked according to the interface predicted aligned error (PAE) score.

- AlphaFold refined ClusPro plus AlphaFold: This pools the AlphaFold-Gap predictions with those of AlphaFold refined ClusPro and re-ranks all 15 predictions using interface PAE.

For ClusPro, AlphaFold refined ClusPro and AlphaFold refined ClusPro plus AlphaFold we use the top 1 numbers from [2]. Figure 1a compares the average DockQ score between the different systems. The AlphaFold-Multimer system has an average DockQ Score of 0.65, while the ClusPro refined AlphaFold system has a score of 0.49 . In order to demonstrate statistical significance, in Figure 1b we compare the relative difference in DockQ score between each system and AlphaFold-Multimer and estimate 95\% confidence intervals using bootstrapping over the targets. A further breakdown of the results, and the per target DockQ scores are provided in Table S1 and Table S2.

\subsection{Recent-PDB-Multimers}

In Figure 2a we show the performance of the system for different levels of similarity to the training set on the Recent-PDB-Multimers dataset. Similarity is defined as identity $\times$ coverage for the least well-matched chain of the pair, maximised over all training set templates for which there is a match for both chains. Here we can see that there is fairly little correlation between similarity to the training set and model quality.

In Figure $2 \mathrm{~b}$ we compare the average DockQ score between AlphaFold-Multimer and AlphaFoldLinker. We see that AlphaFold-Multimer is more accurate on both homomeric and the heteromeric interfaces: in the homomeric case the improvement is relatively modest with +0.05 mean DockQ score, but in the heteromeric case the improvement is more pronounced with +0.19 mean DockQ score. On average the performance on homomeric interfaces is better than for heteromeric ones. In Figure $2 c$ 
we show the difference between the two systems with bootstrapped error bars, showing clearly that AlphaFold-Multimer demonstrates a statistically significant improvement. The proportion of targets above different DockQ scores is shown in Figure 2d, a further breakdown into DockQ categories (Incorrect, Acceptable, Medium and High) is available in Table S3. The AlphaFold-Multimer system successfully predicts (DockQ $\geq 0.23$ ) heteromeric interfaces in $67 \%$ of cases, and successfully predicts homomeric interfaces in $69 \%$ of cases.

In Figure 3 we show the distribution of DockQ score as a function of predicted interface TM-score for the AlphaFold-Multimer system. The figure shows that our predicted accuracy metric is a good predictor of model quality with the majority of high confidence predictions also having high accuracy.

In Figure 4 we show a number of examples where AlphaFold successfully predicted the right multimeric structure. In order to illustrate how to understand the predicted error metric we show an example of a case with incorrect coordinates (PDB code: 6QF7) which is also predicted to be wrong in Figure 5. In this case the individual chains are predicted to be correct, while their predicted relative position is uncertain, as can be seen from the blocks with a high predicted error.

The AlphaFold system was trained to predict the individual chains of multimeric complexes, and as such the model was not given the context provided by the other chains explicitly. Nevertheless AlphaFold has been shown to often implicitly model structures with missing context correctly. In the AlphaFold paper [1] an example is provided of a heavily intertwined multimer with correctly predicted but not physically meaningful chain structure. This leads us to ask the question whether the individual chain predictions of AlphaFold-Multimer are more accurate than the corresponding AlphaFold predictions. In Figure 6 we compare the performance of AlphaFold-Linker to AlphaFold-Multimer under two regimes. In AlphaFold-Multimer the full complex is folded before extracting the individual chains, so that the system sees additional context. In the second regime (AlphaFold-Multimer as Monomers and AlphaFold) the individual chains are folded in isolation. We can see that in the case of heteromers AlphaFold-Multimer is less accurate than AlphaFold when given the single chains with incorrect monomer stoichiometry, but is more accurate than AlphaFold when the single chains are predicted as part of the full complex. We do not observe a similar effect in the AlphaFold-Linker system.

\section{Discussion}

We have shown that by modifying the AlphaFold architecture to natively handle multimers and training explicitly on oligomeric data, we are able to provide high accuracy predictions for a large fraction of PDB complexes, surpassing the accuracy of inference-only modifications to AlphaFold. We have not yet implemented multimer templates or self-distillation of multimer predictions, so there is also likely substantial scope for future accuracy improvements.

We observe that performance is generally higher for homomeric interfaces than for heteromeric interfaces; this is presumably since in the homomeric case the MSA will readily encode evolutionary information about the complex interfaces, while this information is more limited and harder to access in the case of heteromeric interfaces. As a limitation, we observe anecdotally that AlphaFold-Multimer is generally not able to predict binding of antibodies and this remains an area for future work. We have also shown that the confidence metrics provided by the model correlate well with the true accuracy, something that is vital for the useability of a structure prediction model. By allowing accurate prediction of protein complexes we hope that this method will enable biologists to further accelerate the recent progress in structural bioinformatics.

The source code and weights for the trained models will be made available shortly. 


\section{Acknowledgements}

We thank C. Beattie, H. Maclean, A. Huber, C. Meyer, C. Donner, A. Ballard, M. Figurnov, S. Nikolov, Z. Wu, J. Adler, J. Dunger, O. Vinyals, F. Yang and all our colleagues at DeepMind, Google and Alphabet for their encouragement and support. 


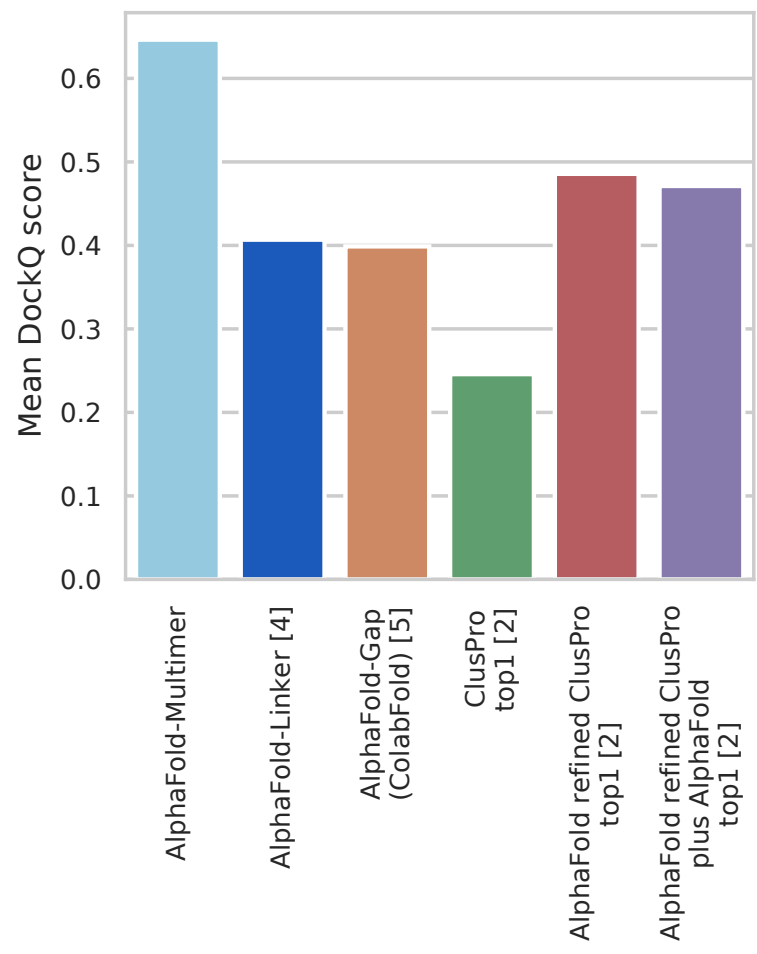

(a)

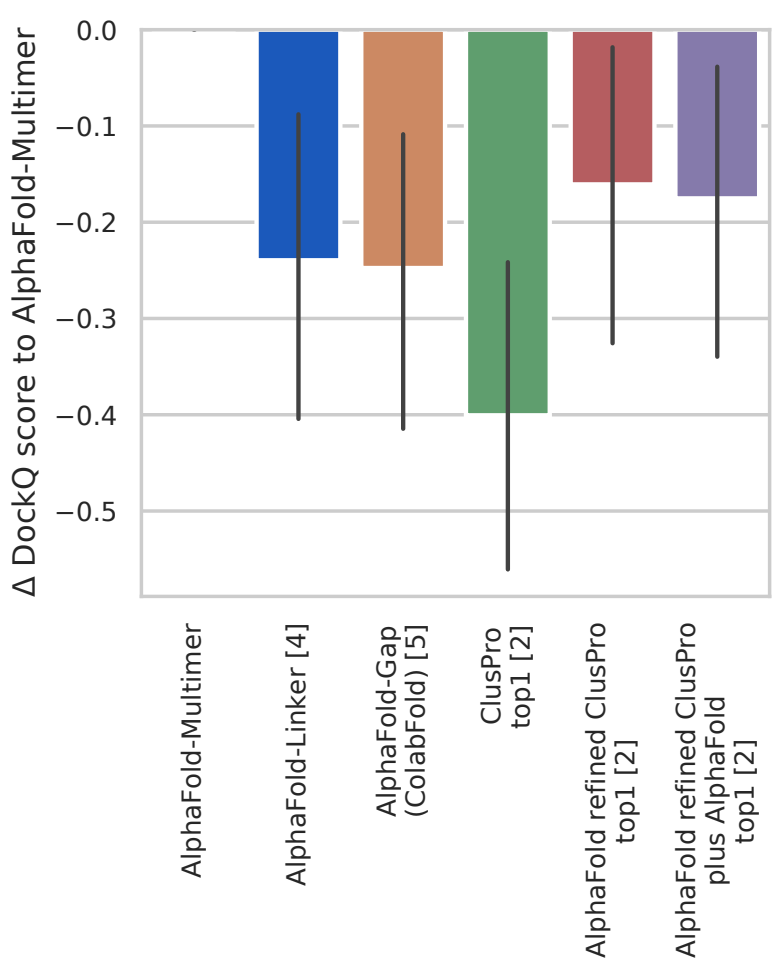

(b)

Figure 1 | The performance of AlphaFold-Multimer against several published baselines is shown on the Benchmark 2 dataset, consisting of 17 heterodimer targets with low training set homology. AlphaFoldLinker is AlphaFold with a 21 residue linker of repeated Glycine-Glycine-Serine residues, similar to previous AlphaFold modifications [4]. AlphaFold-Gap (ColabFold [5]), version from 2021-08-16, is a published system that runs AlphaFold with a gap between residue indices between chains, uses MMSeqs2 for genetics, includes MSA pairing and does not include templates. ClusPro, AlphaFold refined ClusPro, and AlphaFold refined ClusPro plus AlphaFold are all systems and results based on combining the docking algorithm ClusPro with AlphaFold, results are as reported in [2]. Error bars represent a 95\% confidence interval around the mean. 


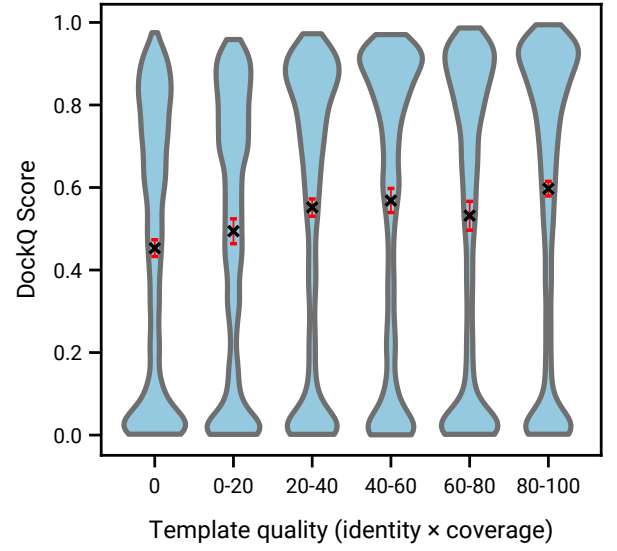

(a)

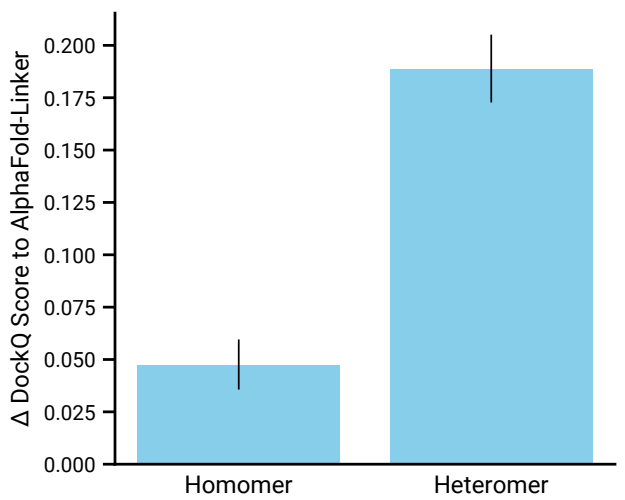

(c)

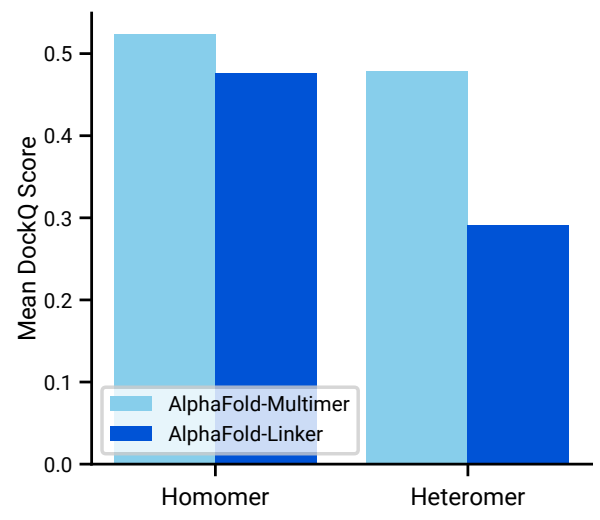

(b)

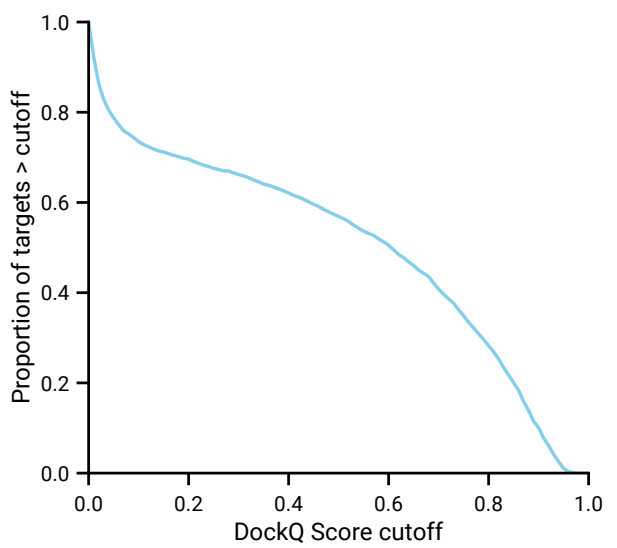

(d)

Figure 2 | The performance of AlphaFold-Multimer on the Recent-PDB-Multimers dataset: the structures of 4,433 non-redundant protein complexes from recent PDB were predicted, from which all non-redundant pairs in contact were extracted (defined as any heavy atom of one chain being within 5A of any heavy atom of the other chain), yielding 5,271 pairs. For figures b, $c$ and $d$ this set was further reduced such that no chain in the pair had greater than $40 \%$ template overlap (identity $\times$ coverage) to the training set, yielding 1,282 homomeric and 1,321 heteromeric scored interfaces. All error bars represent a 95\% confidence interval around the mean. 


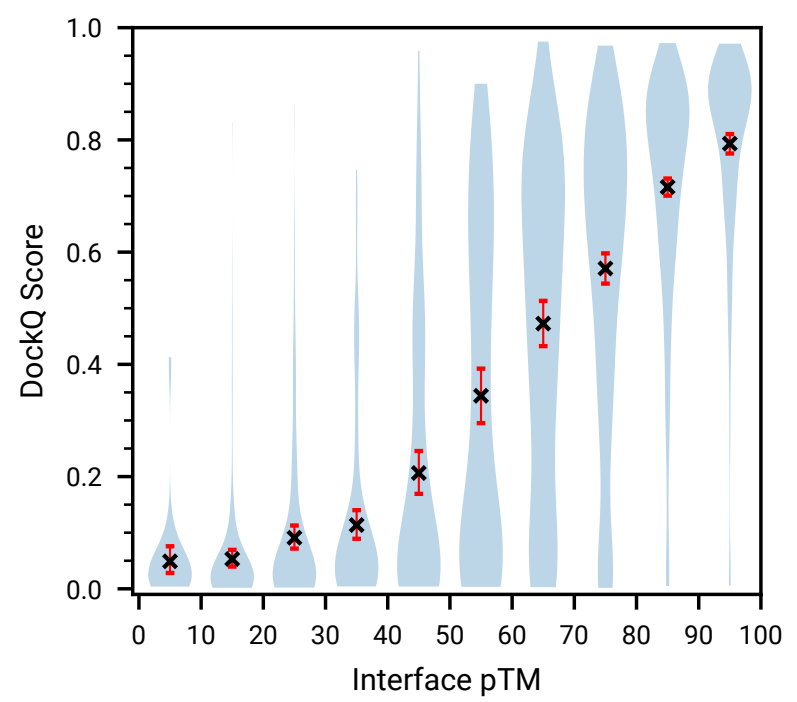

(a)

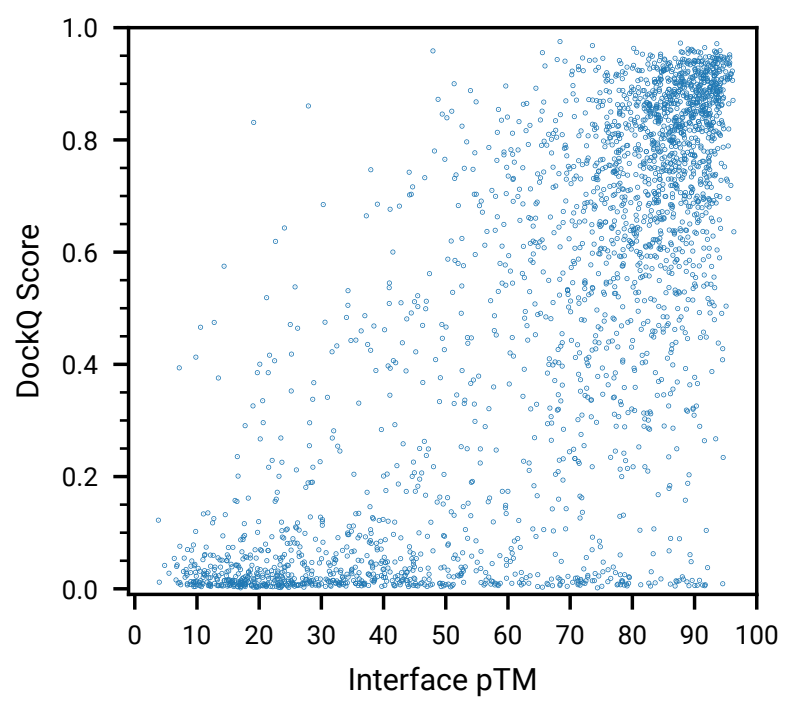

(b)

Figure 3 | The correlation between AlphaFold-Multimer model confidence (predicted interface TM-score) and the true DockQ score is shown for the 2,648 homology reduced in-contact chain pairs from the Recent-PDB-Multimers dataset. Error bars represent a 95\% confidence interval around the mean. In (a) interface PTM is grouped into intervals of size 10, (b) shows the raw data points. 


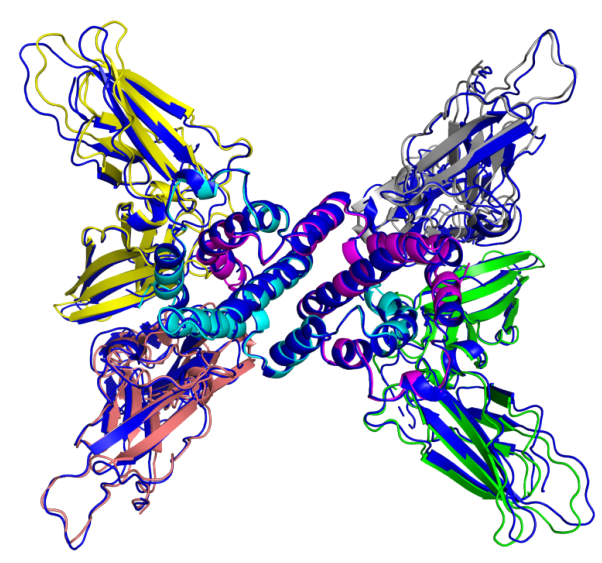

(a) $\mathrm{A} 2 \mathrm{~B} 2 \mathrm{C} 2$ heteromer

TM-score $=98.0, N_{\text {res }}=1,246$, PDB ID $=6 \mathrm{E} 3 \mathrm{~K}$

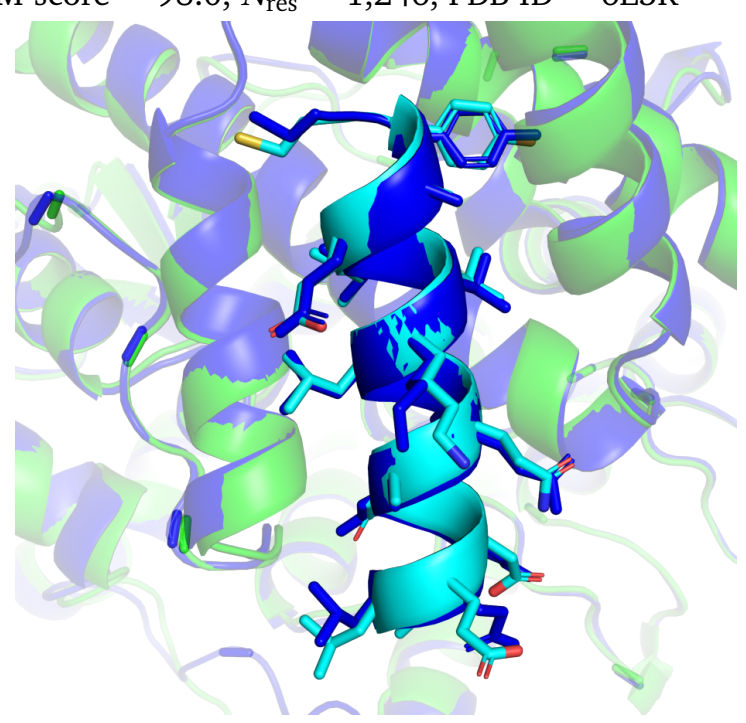

(c) Protein-peptide complex

TM-score $=96.0$, DockQ $=0.948$,

$N_{\text {res }}=385$, PDB ID $=6 \mathrm{JMT}$

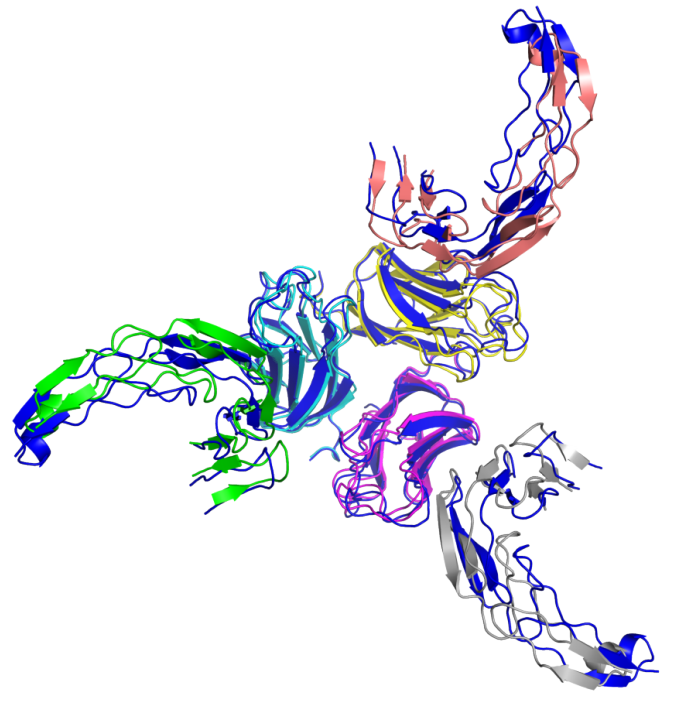

(b) A3B3 heteromer

TM-score $=89.3, N_{\text {res }}=795$, PDB ID $=7 \mathrm{KHD}$

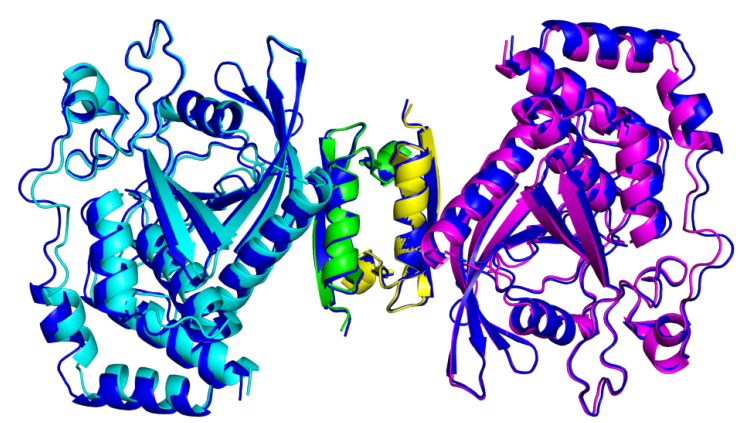

(d) A2B2 heteromer

TM-score $=98.3, N_{\text {res }}=716$, PDB ID $=6$ IWD

Figure 4 | Structure examples predicted with the AlphaFold-Multimer. Visualised are the ground truth structures (blue) and predicted structures (coloured by chain). 

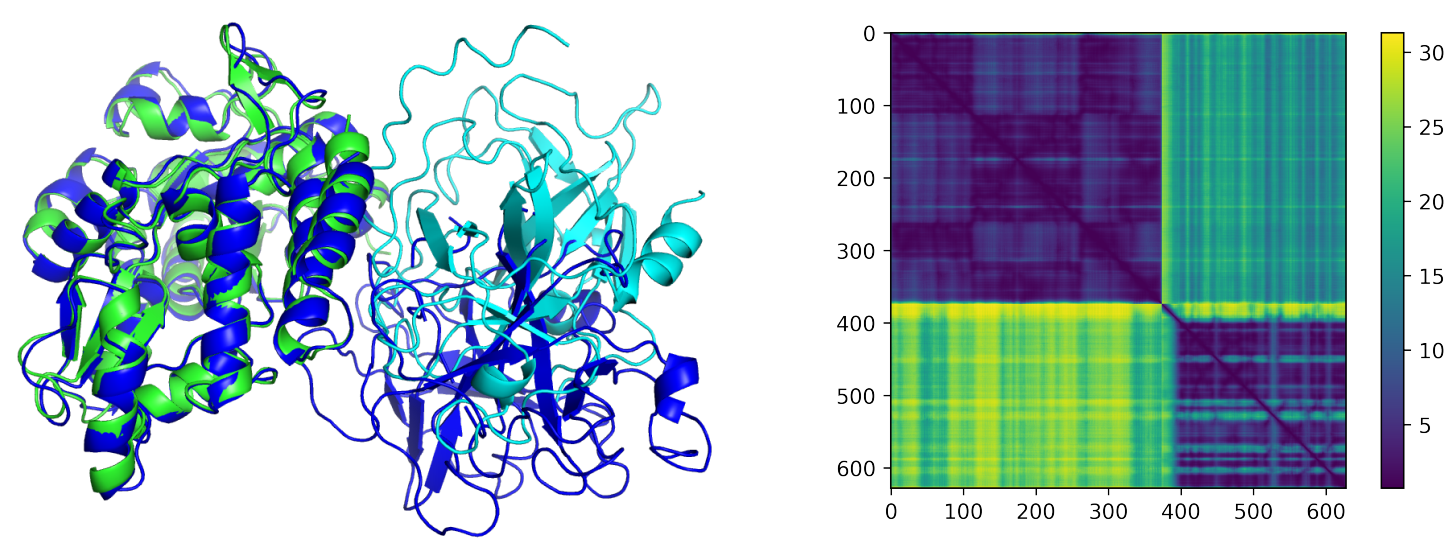

TM-score $=67.4$, DockQ $=0.04, N_{\text {res }}=628, \mathrm{PDB}$ ID $=6 \mathrm{QF7}$

Figure 5 | Example of a predicted heterodimer with incorrect geometry that is correctly predicted as low confidence by the predicted aligned error (PAE). Visualised are the ground truth structures (blue), predicted structures (coloured by chain), and PAE heat map. The PAE heat map shows the predicted error (in Angstroms) between all pairs of residues. 

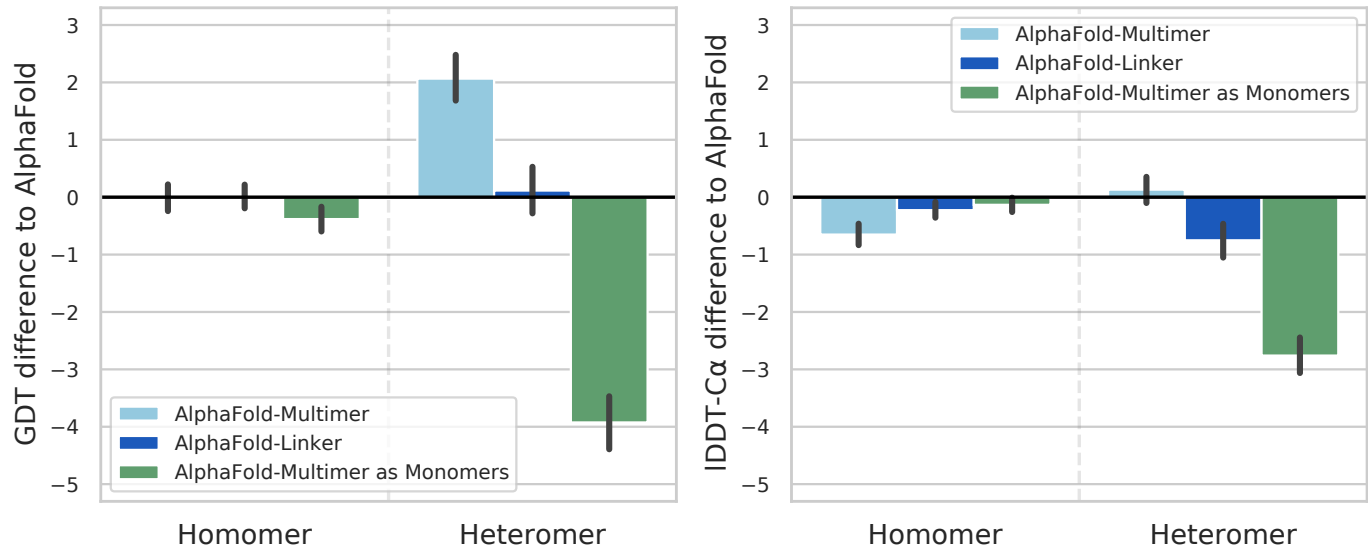

Figure 6 | Comparison on 5,029 clustered single chains extracted from the Recent-PDB-Multimers dataset. Metrics for AlphaFold-Linker and AlphaFold-Multimer are computed by predicting the full-complex, then cropping out the individual chains. For AlphaFold and AlphaFold-Multimer as Monomers the individual chains are run with A1 stoichiometry instead of the true stoichiometry. All error bars represent a 95\% confidence interval around the mean. 


\section{References}

[1] John Jumper, Richard Evans, Alexander Pritzel, Tim Green, Michael Figurnov, Olaf Ronneberger, Kathryn Tunyasuvunakool, Russ Bates, Augustin Žídek, Anna Potapenko, et al. Highly accurate protein structure prediction with AlphaFold. Nature, 596(7873):583-589, 2021.

[2] Usman Ghani, Israel Desta, Akhil Jindal, Omeir Khan, George Jones, Sergey Kotelnikov, Dzmitry Padhorny, Sandor Vajda, and Dima Kozakov. Improved docking of protein models by a combination of AlphaFold2 and ClusPro. bioRxiv, 2021.

[3] Sankar Basu and Björn Wallner. DockQ: a quality measure for protein-protein docking models. PloS one, 11(8):e0161879, 2016.

[4] Yoshitaka Moriwaki (@Ag_smith). Twitter post: AlphaFold2 can also predict heterocomplexes. all you have to do is input the two sequences you want to predict and connect them with a long linker. https://twitter.com/Ag_smith/status/1417063635000598528. 2021-07-19.

[5] Sergey Ovchinnikov, Milot Mirdita, and Martin Steinegger. ColabFold-making protein folding accessible to all via google colab, 2021.

[6] Minkyung Baek (@minkbaek). Twitter post: Adding a big enough number for residue_index feature is enough to model hetero-complex using AlphaFold (green\&cyan: crystal structure / magenta: predicted model w/ residue_index modification). https://twitter.com/minkbaek/status/1417538291709071362. 2021-07-20.

[7] Junsu Ko and Juyong Lee. Can AlphaFold2 predict protein-peptide complex structures accurately? bioRxiv, 2021.

[8] Tomer Tsaban, Julia K Varga, Orly Avraham, Ziv Ben Aharon, Alisa Khramushin, and Ora SchuelerFurman. Harnessing protein folding neural networks for peptide-protein docking. bioRxiv, 2021.

[9] Patrick Bryant, Gabriele Pozzati, and Arne Elofsson. Improved prediction of protein-protein interactions using AlphaFold2 and extended multiple-sequence alignments. bioRxiv, 2021.

[10] AlphaFold open source code. https://github.com/deepmind/alphafold. Accessed: 2021-09-14.

[11] DP Wall, HB Fraser, and AE Hirsh. Detecting putative orthologs. Bioinformatics, 19(13):1710-1711, 2003.

[12] Tian-ming Zhou, Sheng Wang, and Jinbo Xu. Deep learning reveals many more inter-protein residue-residue contacts than direct coupling analysis. bioRxiv, page 240754, 2018.

[13] Stephen K Burley, Charmi Bhikadiya, Chunxiao Bi, Sebastian Bittrich, Li Chen, Gregg V Crichlow, Cole H Christie, Kenneth Dalenberg, Luigi Di Costanzo, Jose M Duarte, et al. RCSB protein data bank: powerful new tools for exploring 3d structures of biological macromolecules for basic and applied research and education in fundamental biology, biomedicine, biotechnology, bioengineering and energy sciences. Nucleic acids research, 49(D1):D437-D451, 2021.

[14] Martin Steinegger and Johannes Söding. Clustering huge protein sequence sets in linear time. Nature communications, 9(1):1-8, 2018.

[15] Aysam Guerler, Brandon Govindarajoo, and Yang Zhang. Mapping monomeric threading to proteinprotein structure prediction. Journal of chemical information and modeling, 53(3):717-725, 2013. 
[16] Rong Chen, Li Li, and Zhiping Weng. Zdock: an initial-stage protein-docking algorithm. Proteins: Structure, Function, and Bioinformatics, 52(1):80-87, 2003.

[17] Dima Kozakov, David R Hall, Bing Xia, Kathryn A Porter, Dzmitry Padhorny, Christine Yueh, Dmitri Beglov, and Sandor Vajda. The ClusPro web server for protein-protein docking. Nature protocols, 12(2):255-278, 2017.

[18] Mieczyslaw Torchala, Iain H Moal, Raphael AG Chaleil, Juan Fernandez-Recio, and Paul A Bates. Swarmdock: a server for flexible protein-protein docking. Bioinformatics, 29(6):807-809, 2013.

[19] Minkyung Baek, Ivan Anishchenko, Hahnbeom Park, Ian R Humphreys, and David Baker. Protein oligomer modeling guided by predicted inter-chain contacts in CASP14. Proteins: Structure, Function, and Bioinformatics, 2021.

[20] Justas Dapkunas, Kliment Olechnovič, and Česlovas Venclovas. Modeling of protein complexes in CASP14 with emphasis on the interaction interface prediction. Authorea Preprints, 2021.

[21] CASP14 abstract book. https://predictioncenter.org/casp14/doc/CASP14_Abstracts.pdf. Accessed: 2021-09-14.

[22] Minkyung Baek, Frank DiMaio, Ivan Anishchenko, Justas Dauparas, Sergey Ovchinnikov, Gyu Rie Lee, Jue Wang, Qian Cong, Lisa N Kinch, R Dustin Schaeffer, et al. Accurate prediction of protein structures and interactions using a 3-track network. bioRxiv, 2021.

[23] Ilya A Vakser. Evaluation of gramm low-resolution docking methodology on the hemagglutininantibody complex. Proteins: Structure, Function, and Bioinformatics, 29(S1):226-230, 1997.

[24] Ian R. Humphreys, Jimin Pei, Minkyung Baek, Aditya Krishnakumar, Ivan Anishchenko, Sergey Ovchinnikov, Jing Zhang, Travis J. Ness, Sudeep Banjade, Saket Bagde, Viktoriya G. Stancheva, Xiao-Han Li, Kaixian Liu, Zhi Zheng, Daniel J. Barrero, Upasana Roy, Israel S. Fernández, Barnabas Szakal, Dana Branzei, Eric C. Greene, Sue Biggins, Scott Keeney, Elizabeth A. Miller, J. Christopher Fromme, Tamara L. Hendrickson, Qian Cong, and David Baker. Structures of core eukaryotic protein complexes. bioRxiv, 2021.

[25] Thomas A Hopf, Charlotta PI Schärfe, João PGLM Rodrigues, Anna G Green, Oliver Kohlbacher, Chris Sander, Alexandre MJJ Bonvin, and Debora S Marks. Sequence co-evolution gives 3d contacts and structures of protein complexes. Elife, 3:e03430, 2014.

[26] Sergey Ovchinnikov, Hetunandan Kamisetty, and David Baker. Robust and accurate prediction of residue-residue interactions across protein interfaces using evolutionary information. elife, 3:e02030, 2014.

[27] Qian Cong, Ivan Anishchenko, Sergey Ovchinnikov, and David Baker. Protein interaction networks revealed by proteome coevolution. Science, 365(6449):185-189, 2019.

[28] Anna G Green, Hadeer Elhabashy, Kelly P Brock, Rohan Maddamsetti, Oliver Kohlbacher, and Debora S Marks. Proteome-scale discovery of protein interactions with residue-level resolution using sequence coevolution. bioRxiv, page 791293, 2019.

[29] Hong Zeng, Sheng Wang, Tianming Zhou, Feifeng Zhao, Xiufeng Li, Qing Wu, and Jinbo Xu. Complexcontact: a web server for inter-protein contact prediction using deep learning. Nucleic acids research, 46(W1):W432-W437, 2018.

[30] DockQ score author's implementation. https://github.com/bjornwallner/DockQ/blob/ 3735c160050f1e9128d2ccb23a0a1945aa98b5b2/DockQ.py. Accessed: 2021-09-14. 
[31] Alex L Mitchell, Alexandre Almeida, Martin Beracochea, Miguel Boland, Josephine Burgin, Guy Cochrane, Michael R Crusoe, Varsha Kale, Simon C Potter, Lorna J Richardson, et al. Mgnify: the microbiome analysis resource in 2020. Nucleic acids research, 48(D1):D570-D578, 2020.

[32] Yang Zhang and Jeffrey Skolnick. Tm-align: a protein structure alignment algorithm based on the tm-score. Nucleic acids research, 33(7):2302-2309, 2005.

[33] Martino Bertoni, Florian Kiefer, Marco Biasini, Lorenza Bordoli, and Torsten Schwede. Modeling protein quaternary structure of homo-and hetero-oligomers beyond binary interactions by homology. Scientific reports, 7(1):1-15, 2017.

[34] L Steven Johnson, Sean R Eddy, and Elon Portugaly. Hidden markov model speed heuristic and iterative hmm search procedure. BMC bioinformatics, 11(1):1-8, 2010.

[35] The UniProt Consortium. UniProt: the universal protein knowledgebase in 2021. Nucleic Acids Research, 49(D1):D480-D489, 2021.

[36] Conrad L Schoch, Stacy Ciufo, Mikhail Domrachev, Carol L Hotton, Sivakumar Kannan, Rogneda Khovanskaya, Detlef Leipe, Richard Mcveigh, Kathleen O'Neill, Barbara Robbertse, et al. NCBI taxonomy: a comprehensive update on curation, resources and tools. Database, 2020, 2020. 


\section{Supplementary Information}

\subsection{Data}

This section describes the AlphaFold-Multimer data pipeline, which generates features for the model. The input to the data pipeline is an mmCIF file containing one or more chains. The AlphaFold-Multimer data pipeline follows similar steps to the single-chain AlphaFold system [1], with a few key differences that are described below.

We derive the details about the structure's biological assemblies from the mmCIF file and uniformly sample a single bio-assembly from all the available bio-assemblies. The MSA features are merged using the cross-genetics method outlined in subsection 7.4.

The AlphaFold-Multimer system uses only per-chain templates. The template search is similar to the AlphaFold system template search, except that it uses HMMER suite v3.3 hmmsearch and hmmbuild instead of HHsearch. First, the Uniref90 MSA obtained in the MSA search is converted to an HMM using hmmbuild (the only flag set to a non-default value is -hand). HMMER hmmsearch is then used to search for matches of the HMM against pdb_seqres.txt, downloaded from ftp://ftp.wwpdb.org/pub/pdb/derived_data/pdb_ seqres.txt on 2020-05-14. We limit the number of templates to 20. Further processing is as described in the AlphaFold paper. Any structure released after 2018-04-30 is excluded from training. The HMMSearch flags used are -F1 0.1 -F2 0.1 -F3 0.1 -incE 100 -E 100 -domE 100 -incdomE 100.

The training data rebalancing procedure is very similar to the AlphaFold single chain setup: we uniformly sample a chain cluster, then uniformly sample a chain within that cluster and select the mmCIF to which it belongs as input to the pipeline. The chain clusters are $40 \%$ identity clusterings of the Protein Data Bank with MMSeqs2 [14].

During the training procedure we use a different self-distillation set, set of filters, MSA preprocessing, and residue cropping methods from the AlphaFold data pipeline. The self-distillation set is created using the clustered MGnify dataset [31], filtered to clusters with more than 10 sequences, resulting in about $13 \mathrm{M}$ sequences.

We filter out proteins during training if:

- The input mmCIF has resolution greater than $9 \AA$.

- Any single amino acid accounts for more than $80 \%$ of the complex sequences.

- The example comes from the distillation set and has less than 200 residues.

Features of the individual chains are merged before further processing. For all identical chains, the features are concatenated along the residue dimension. $N_{\text {res }} \times N_{\text {seq }}$ features of different chains are paired using the cross-chain genetics method outlined in subsection 7.4. $N_{\text {res }}$ features were simply concatenated along the residue dimension.

The merged features are processed in a similar manner to AlphaFold, including MSA sampling and clustering. MSA block deletion is not included in the AlphaFold-Multimer data pipeline.

The features input to the multimer model are identical to the AlphaFold with one extra feature: asym_id, an integer indicating the chain number. The ordering of the input chains is arbitrary as the network is agnostic to chain order. 


\subsection{Multi-chain Cropping}

\subsubsection{Contiguous Cropping}

In this method (Algorithm 1), we iterate through the list of chains, selecting a contiguous crop from each until we have reached our $N_{\text {res }}$ budget. We keep track of two variables during the procedure: $n_{\text {added }}$ is the number of residues selected so far, and $n_{\text {remaining }}$ is the combined length of yet to be cropped chains (excluding the current chain). crop_size_min taken for each chain ensures we use as much of our $N_{\text {res }}$ budget as possible, and crop_size_max ensures we don't go over it. The input to the algorithm is the set of chain lengths, $\left\{n_{k}\right\}$, and the total residue budget $N_{\text {res }}$. The output is a set of masks to apply to each chain in order to extract the corresponding crops, $\left\{\mathrm{m}_{k}\right\}$. The chains are first randomly shuffled to avoid bias.

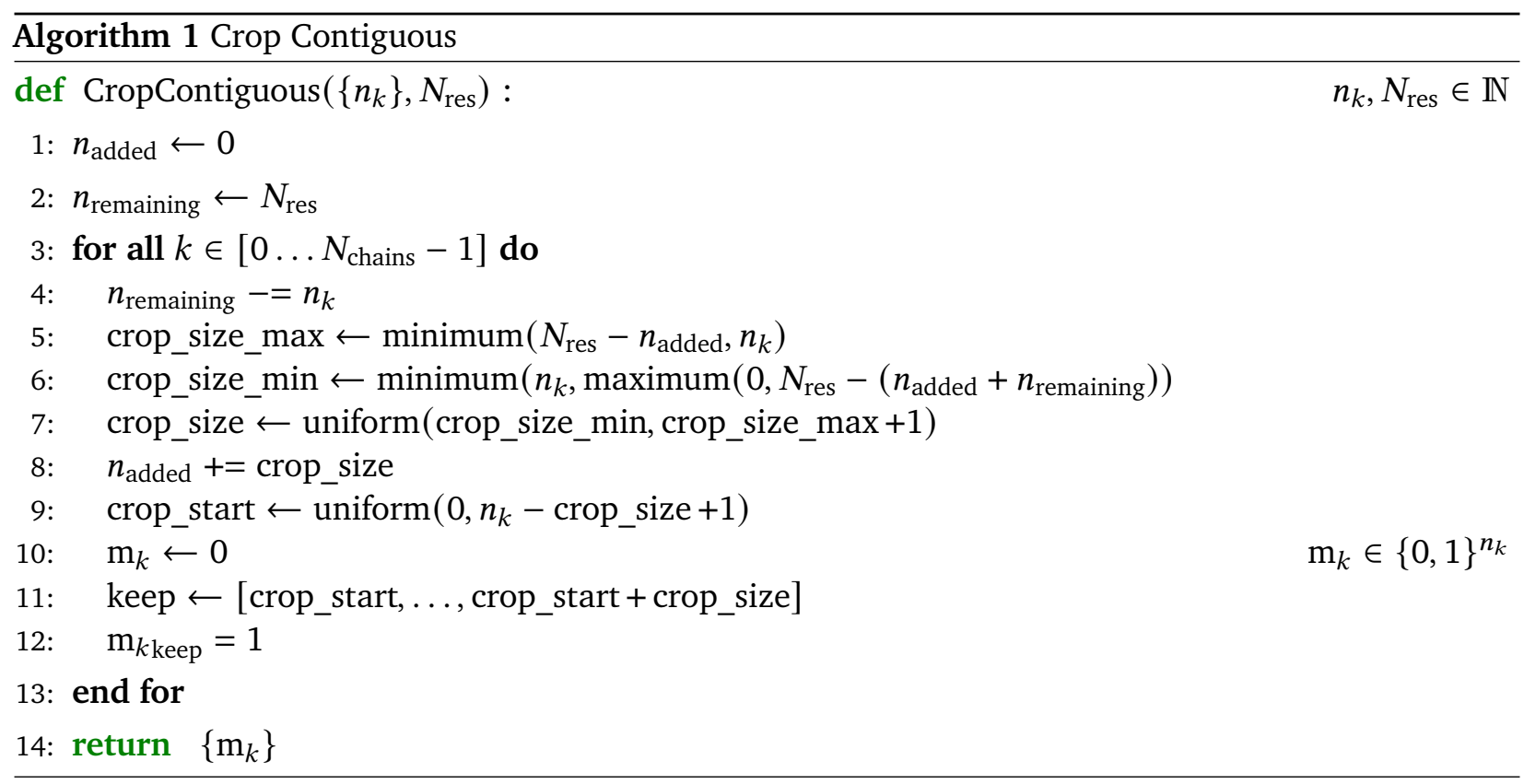

\subsubsection{Spatial Cropping (Interface Biased)}

In this method (Algorithm 2), we specifically target interface regions by selecting a 3D spatial crop around a randomly selected interface residue. The crop consists of the $N_{\text {res }}$ spatially nearest neighbours to the selected interface residue, as defined by distances between $\mathrm{C} \alpha$ coordinates. The input to the algorithm is the set of $\mathrm{C} \alpha$ coordinates across all chains, $\left\{\mathrm{x}_{i}\right\}$, and the index of the selected interface residue, $c$. The output is a set of binary values indicating which residues to include in the crop, $\left\{m_{i}\right\}$. Distance ties are broken by adding small uniquifying values. We found empirically that a 50:50 ratio of interface cropping to contiguous cropping worked best for multimers (always contiguous cropping for monomers).

\subsection{Multi-Chain Permutation Alignment}

Stoichiometry must be carefully accounted for when scoring multimer structure predictions. In a prediction for A2B, both orderings of the A chains are equally valid, regardless of their ordering in the ground truth. In particular, if this is not accounted for in the loss then correct predictions will be unfairly penalized and the network will fail to train properly.

To address this issue, before scoring a multimer prediction we first permute chains with identical 


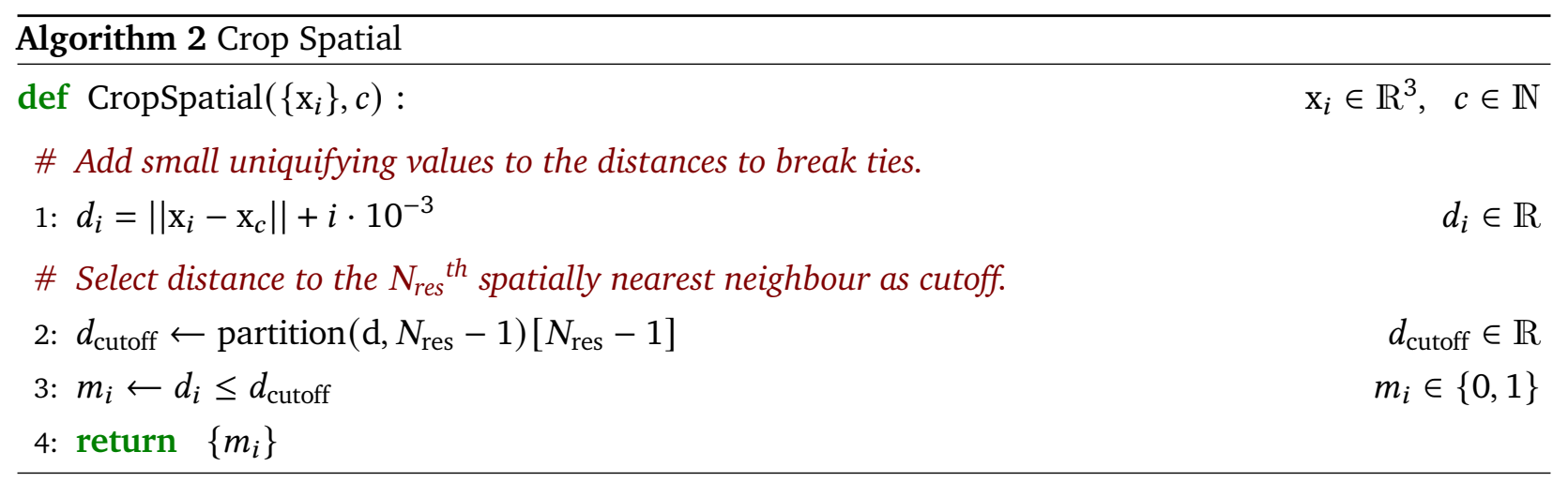

sequences such that they are best-effort aligned with those of the prediction. One could imagine considering the best alignment over all possible permutations, however this quickly becomes intractable, so a heuristic method is needed. Our method is a simple greedy heuristic that can be performed efficiently on TPU; other multi-chain alignment algorithms also exist $[32,33]$. The optimal ground truth chain permutation is found using Algorithm 3, which can be broken down into the following alignment and assignment stages.

\subsubsection{Alignment Stage}

In the alignment phase, we pick a pair of anchor chains to align, one in the ground truth and one in the prediction. The ground truth anchor chain $a^{\text {gt }}$ is chosen to be the least ambiguous possible, for example in an A3B2 complex an arbitrary B chain is chosen. In the event of a tie e.g. A2B2 stoichiometry, the longest chain is chosen, with the hope that in general the longer chains are likely to have higher confident predictions. The prediction anchor chain is chosen from the set $\left\{a_{m}^{\text {pred }}\right\}$ of all prediction chains with the same sequence as the ground truth anchor chain. We then transform the ground truth chain $\mathrm{C} \alpha$ coordinates $\left\{\mathrm{x}_{l}^{\mathrm{gt}}\right\}$ onto the prediction chain $\mathrm{C} \alpha$ coordinates $\left\{\mathrm{x}_{k}^{\text {pred }}\right\}$ such that the chosen pair of anchor chains is optimally aligned (line 2).

\subsubsection{Assignment Stage}

In the assignment stage (Algorithm 4), we greedily assign each of the predicted chains to their nearest neighbour of the same sequence in the ground truth. These assignments define the optimal permutation to apply to the ground truth chains. Nearest neighbours are defined as the chains with the smallest distance between the average of their $\mathrm{C} \alpha$ coordinates.

We repeat the above alignment and assignment stages for all valid choices of the prediction anchor chain $\left\{a_{m}^{\text {pred }}\right\}$ given the ground truth anchor chain $a^{\text {gt }}$. Finally, we pick the permutation (line 8) that minimizes the RMSD between the $\mathrm{C} \alpha$ coordinate averages of the predicted and ground truth chains.

The above procedure must be modified during training to account for cropping. In particular, in the alignment stage, we must first restrict the ground truth anchor chain to the same crop region as the paired prediction chain before alignment. Similarly, in the assignment stage, for each predicted chain the ground truth chains must be restricted to the same crop region before assignment. 

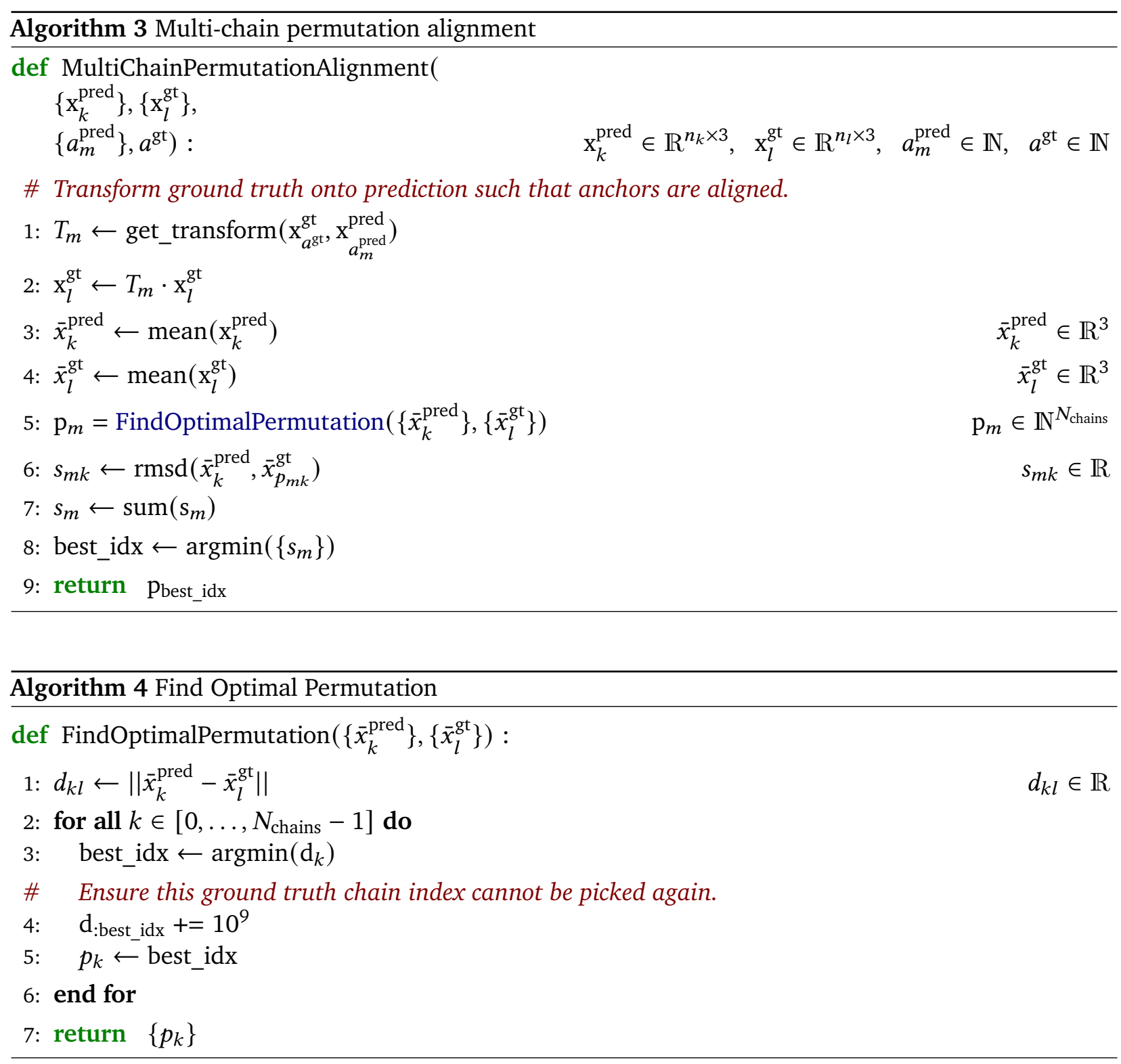

\subsection{Cross-Chain Genetics}

In order to make cross-chain coevolutionary information available to the network, it is necessary to pair sequences between the per-chain MSAs such that they form valid orthologs of the target sequence. In practice, this pairing can't be done exactly, and various heuristic methods are used instead to approximate it. We follow the procedure of Zhou et al. [12], which pairs sequences of the same species and disambiguates according to either genetic distance or sequence similarity, depending on whether the species is prokaryotic or eukaryotic. Our exact steps are as follows:

1. For each chain, JackHMMER v3.3 [34] is used to query the unclustered UniProt 2020_05 database [35] with maximum MSA depth set to 50,000, and non-default flags values set to $-\mathrm{N} 1$-e 0.0001 -Z 119222328.

2. The per-chain MSA sequences are grouped by species, using species labels derived from UniProt's idmapping downloaded from https://ftp.uniprot.org/pub/databases/uniprot/current_release/knowledgebase/idmapping/ for the 2020_05 UniProt version. 
3. Sequences are then paired within a specific species. If there is only one sequence per species, we simply concatenate them. Otherwise, we need to disambiguate. The disambiguation method differs depending on whether the species is eukaryotic or prokaryotic.

- Prokaryote: we match the chain MSAs by minimizing the base-pair distance between chains.

(a) The chains are considered in order of the chain id. Candidate MSA hits from the first chain are considered in order of e-value provided by JackHMMER.

(b) Hits for second and subsequent chains are chosen to minimize the base-pair distance from the previous chain's hit, with a hard cut-off if the distance is too great. Base-pair distance is approximated using the lexicographic difference of the Uniprot accession IDs.

(c) If a candidate MSA hit has more than $90 \%$ sequence identity to the query chain, or more than $50 \%$ unmatched gaps, the entire matched set of hits up to that chain is discarded and we move onto the next candidate MSA hit from the first chain.

- Eukaryote: we match the chain MSAs by ordering them by sequence identity to the target sequence and matching sequences of the same rank. There will be as many matches as there are hits in the shortest MSA.

4. In both cases, chains with empty MSAs are not matched and the resulting paired MSA will be padded with gap (no-match) symbols.

5. The target complex is identified as eukaryotic or prokaryotic from the PDB entry for the complex, using the NCBI taxonomy database [36] and UniProt taxonomy [35] to determine the domain. We default to the eukaryotic method where the domain is unknown.

\subsection{Chain Relative Positional Encoding}

In AlphaFold [1], relative positional features are encoded into the initial pair representation of the network, allowing the network to reason about the positions of residues in the chain and break symmetry between identical residues. In the case of multimers, we also need to tell the network when two residues are in different chains, allowing it to break symmetry between homomer chains. To do this (Algorithm 5) we simply add an extra bin to the relative position encoding $f_{i j}^{\text {rel pos }}$ of AlphaFold to denote 'different chain'. Furthermore, we also provide a feature to the network $f_{i j}^{\text {same_entity }}$ encoding whether the residues are from chains with identical sequences, and another feature $f_{i j}^{\text {rel }}$ chain encoding relative chain index between chains of identical sequence. Relative residue indices are clipped between $\left[-r_{\max }, r_{\max }\right]$, with $r_{\max }=32$, and relative chain indices are clipped between $\left[-s_{\max }, s_{\max }\right]$, with $s_{\max }=2$. $f_{i}^{\text {residue }{ }_{-} \text {index }}$ is the index of residue $i$, and $f_{i}^{\text {asym_id }}, f_{i}^{\text {entity_id }}$ and $f_{i}^{\text {sym_id }}$ are chain identifiers for residue $i$ : $f_{i}^{\text {asym_id }}$ distiguishes between chains, $f_{i}^{\text {entity_id }}$ distinguishes between unique chain sequences, and $f_{i}^{\text {sym_id }}$ distinguishes between chains of the same sequence.

\subsection{Mixed Frame Aligned Point Error (FAPE) Clamping}

In AlphaFold the frame aligned point error (FAPE) backbone loss is clamped at $10 \AA$, emphasizing accurate positioning of residues within their local neighbourhood, but de-emphasizing longer range errors. For multimer chains, we also want to encourage accurate positioning of interface residues, which are in general much farther apart than neighbouring within-chain residues. We achieve this by splitting the FAPE loss into two. The first is clamped at $10 \AA$ and applied to within-chain residue-pairs; the second is unclamped and applied to between-chain residue-pairs. These two losses are then summed (which also has the effect of upweighting between-chain residue-pairs). 

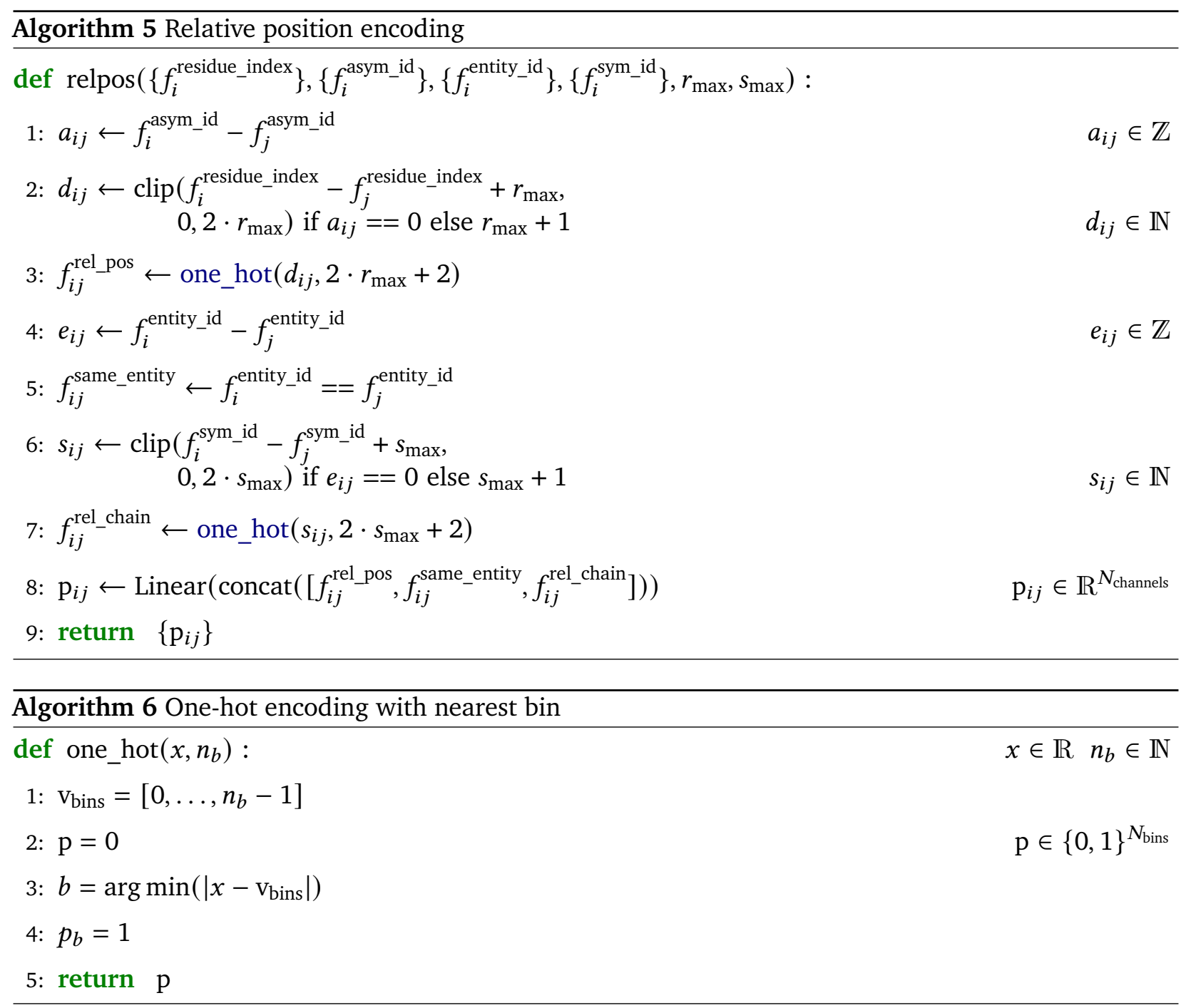

\subsection{Architectural Modifications}

We made various small architecture changes to facilitate inferencing proteins of larger size, and made some further minor changes to make different components of the model more consistent with one another.

We swap the order of the attention and triangular multiplicative update layers in the template stack relative to the AlphaFold model. This is to make it consistent with the order in the Evoformer stack. The template unit vectors that were disabled in the AlphaFold model are enabled here, i.e. they are not set to zero but computed from the template coordinates. We changed the way the template embeddings are aggregated. Before the model would give a pairwise representation of embeddings for each template, which were then aggregated via attention independently for each pair of residues. Here we use a simpler approach whereby we simply average the template embeddings. For efficiency we compute this similarly to how a moving average would be computed: we sum the embeddings as they are getting produced and divide by number of templates at the end. This has the advantage that it does not require template embeddings for all templates in order to average them, which leads to a substantial reduction in memory consumption. We have observed no significant drop in accuracy when aggregating templates in this way. Furthermore we moved the outer product mean to the start of the Evoformer block. This allows the pair representation and the MSA representation to evolve independently within a given block, with all cross 
communication happening at the start of the block. At training time this means that we do not need to keep all of the MSA stack activations and all the pair stack activations in memory at the same time during backpropagation. At inference time both stacks can be processed in parallel.

\subsection{Datasets}

The Recent-PDB-Multimers set consists of all targets in the Protein Data Bank [13] released between 2018-04-30 and 2021-08-02. This set was filtered to proteins with more than one chain, less than 9 chains and less than 1,536 total residues. It was also clustered with the following approach, which yields a set of 4,433 protein complexes:

1. Assign each chain to its $40 \%$ overlap cluster (using the clusters provided by PDB (link)).

2. Assign each protein complex a cluster identifier that is the union of all the single-chain cluster ids from step 1.

3. Randomly pick a single protein complex from every full-complex cluster.

Predictions were made on the full complexes, however for Recent-PDB-Multimers metrics were computed for each chain-pair separately. The chain pairs were selected if they were 'in contact' in the ground truth, defined as any heavy atom of one chain being within 5A of any heavy atom of the other chain. They were then clustered to remove redundancy: chain pairs were greedily selected if the cluster id pair was unique amongst those already selected. Finally the data was filtered such that no chain has greater than $40 \%$ template identity to the training set. This resulted in 2,603 unique in-contact chain-pairs $(5,271$ pre-filtering).

\subsection{Interface pTM}

Defining an interface version of AlphaFold's predicted TM-score metric [1, §1.9.7] amounts to modifying [1, Equation 40] so that $i$ and $j$ come from different chains.

Concretely, if we follow the notation in [1, §1.9.7] such that $X^{\text {true }}=\left\{\vec{x}_{j}^{\text {true }}\right\}, X=\left\{\vec{x}_{j}\right\}$ are the $\mathrm{C} \alpha$ positions of the ground truth and predicted structures respectively, $\left\{T_{i}^{\text {true }}\right\},\left\{T_{i}\right\}$ are the corresponding backbone frames, $e_{i j}=\left\|T_{i}^{-1} \circ \vec{x}_{j}-T_{i}^{\text {true }}{ }^{-1} \circ \vec{x}_{j}^{\text {true }}\right\|$ is the error in the position of the $\mathrm{C} \alpha$ atom of residue $j$ when the predicted and true structures are aligned using the backbone frame of residue $i, N_{\text {res }}$ is the total number of residues, and $d_{0}\left(N_{\text {res }}\right)=1.24 \sqrt[3]{\operatorname{maximum}\left(N_{\text {res }}, 19\right)-15}-1.8$ is the TM-score normalization constant, then we can obtain a interface TM-score prediction as:

$$
\operatorname{ipTM}=\max _{i} \frac{1}{\left|\mathcal{D}_{- \text {chain }(i)}\right|} \sum_{j \in \mathcal{D}_{- \text {chain }(i)}} \mathbb{E} \frac{1}{1+\left(\frac{e_{i j}}{d_{0}\left(\left|\mathcal{D}_{- \text {chain }(i)}\right|\right)}\right)^{2}},
$$

where $\mathcal{D}_{- \text {chain }(i)}$ is the set of all residues except those of the chain of residue $i$. As in [1, \$1.9.7], the expectation is taken over the probability distribution defined by $e_{i j}$, which is predicted by a neural network. 


\begin{tabular}{lcrrrrr}
\hline Method & $\begin{array}{c}\text { Mean } \\
\text { DockQ } \\
\text { Score }\end{array}$ & $\begin{array}{c}\text { Incorrect } \\
\text { Count }\end{array}$ & $\begin{array}{c}\text { Acceptable } \\
\text { Count }\end{array}$ & $\begin{array}{c}\text { Medium } \\
\text { Count }\end{array}$ & $\begin{array}{c}\text { High } \\
\text { Count }\end{array}$ \\
\hline AlphaFold-Multimer & 0.65 & 3 & 0 & 8 & 6 \\
$\begin{array}{c}\text { AlphaFold refined ClusPro } \\
\text { AlphaFold refined ClusPro }\end{array}$ & 0.49 & 7 & 1 & 4 & 5 \\
$\quad 0.47$ & 7 & 1 & 5 & 4 \\
$\quad$ plus AlphaFold & 0.41 & 7 & 1 & 5 & 4 \\
AlphaFold-Linker & 0.40 & 8 & 1 & 6 & 2 \\
ClusPro & 0.25 & 10 & 3 & 4 & 0 \\
\hline
\end{tabular}

Table S1 | Performance on the Benchmark 2 dataset from [2] consisting of 17 heterodimers with low training set similarity. The following CAPRI definitions were used:

Incorrect: $0 \leq$ DockQ $<0.23$

Acceptable: $0.23 \leq$ DockQ $<0.49$

Medium: $0.49 \leq$ DockQ $<0.80$

High: $0.80 \leq$ DockQ

\begin{tabular}{lr}
\hline Target & DockQ Score \\
\hline 5ZNG & 0.02 \\
6A6I & 0.05 \\
6GS2 & 0.50 \\
6H4B & 0.80 \\
6IF2 & 0.74 \\
6II6 & 0.73 \\
6ONO & 0.64 \\
6PNQ & 0.56 \\
6Q76 & 0.91 \\
6U08 & 0.93 \\
6ZBK & 0.79 \\
7AYE & 0.88 \\
7D2T & 0.78 \\
7M5F & 0.89 \\
7N10 & 0.86 \\
7NLJ & 0.06 \\
7P8K & 0.86 \\
\hline
\end{tabular}

Table S2 | Results per target for AlphaFold-Multimer, on the 17 heterodimer Benchmark 2 dataset from [2] 
(a) Homomeric Interfaces

\begin{tabular}{lrrrrr}
\hline Method & $\begin{array}{c}\text { Mean } \\
\text { DockQ } \\
\text { Score }\end{array}$ & Incorrect \% & Acceptable \% & Medium \% & High \% \\
\hline AlphaFold-Linker & 0.476 & 35.7 & 10.1 & 25.4 & 28.8 \\
AlphaFold-Multimer & 0.523 & 30.7 & 9.83 & 25.1 & 34.3 \\
\hline
\end{tabular}

(b) Heteromeric Interfaces

\begin{tabular}{lcrrrr}
\hline Method & $\begin{array}{c}\text { Mean } \\
\text { DockQ } \\
\text { Score }\end{array}$ & Incorrect \% & Acceptable \% & Medium \% & High \% \\
\hline AlphaFold-Linker & 0.290 & 57.5 & 10.4 & 20.6 & 11.4 \\
AlphaFold-Multimer & 0.479 & 32.7 & 11.9 & 33.1 & 22.3 \\
\hline
\end{tabular}

Table S3 | Performance on the Recent-PDB-Multimers dataset, evaluated on homology-reduced chain pairs with low training set similarity (see subsection 7.8), broken down into DockQ categories as in Table S1. 